\title{
ARTIFICIAL INTELLIGENCE IN PREDICTING HEART FAILURE
}

\author{
Rashid Ebrahim Al-Mannai ${ }^{1}$, Mohammed Hamad Almerekhi ${ }^{1}$, Mohammed Abdulla Al-Mannai ${ }^{1}$, Mishahira ${ }^{2}$, Kishor Kumar \\ Sadasivuni2*, Huseyin Cagatay Yalcin ${ }^{3}$, Hassen M. Ouakad ${ }^{*}$,Issam Bahadur ${ }^{5}$, Somaya Al-Maadeed ${ }^{1}$,Asiya Albusaidi ${ }^{4}$ \\ ${ }^{1}$ Department of Computer Science and Engineering, Qatar University, Qatar. ${ }^{2}$ Center for Advanced Materials, Qatar University, Qatar. \\ ${ }^{3}$ Biomedical Research Center, Qatar University, Qatar. ${ }^{4}$ Mechanical and Industrial Engineering Department, College of Engineering, \\ 5Sultan Qaboos University, Muscat, Oman.
}

Undergraduate Students, Health and Biomedical Science

\section{Kishorkumars@qu.edu.qa $\underline{\text { houakad@squ.edu.om }}$}

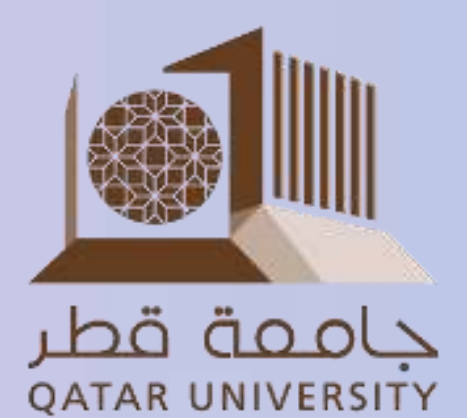

\begin{abstract}
Heart Failure is a major chronic disease that is increasing day by day and a great health burden in health care systems worldwide. Artificial intelligence $(\mathrm{Al})$ techniques such as machine learning $(\mathrm{ML})$, deep learning (DL), and cognitive computer can play a critical role in the early detection and diagnosis of Heart Failure Detection, as well as outcome prediction and prognosis evaluation. The availability of large datasets from difference sources can be leveraged to build machine learning models that can empower clinicians by providing early warnings and insightful information on the underlying conditions of the patients.

Keywords: Heart Failure; Machine learning; Supervised learning; unsupervised learning
\end{abstract}
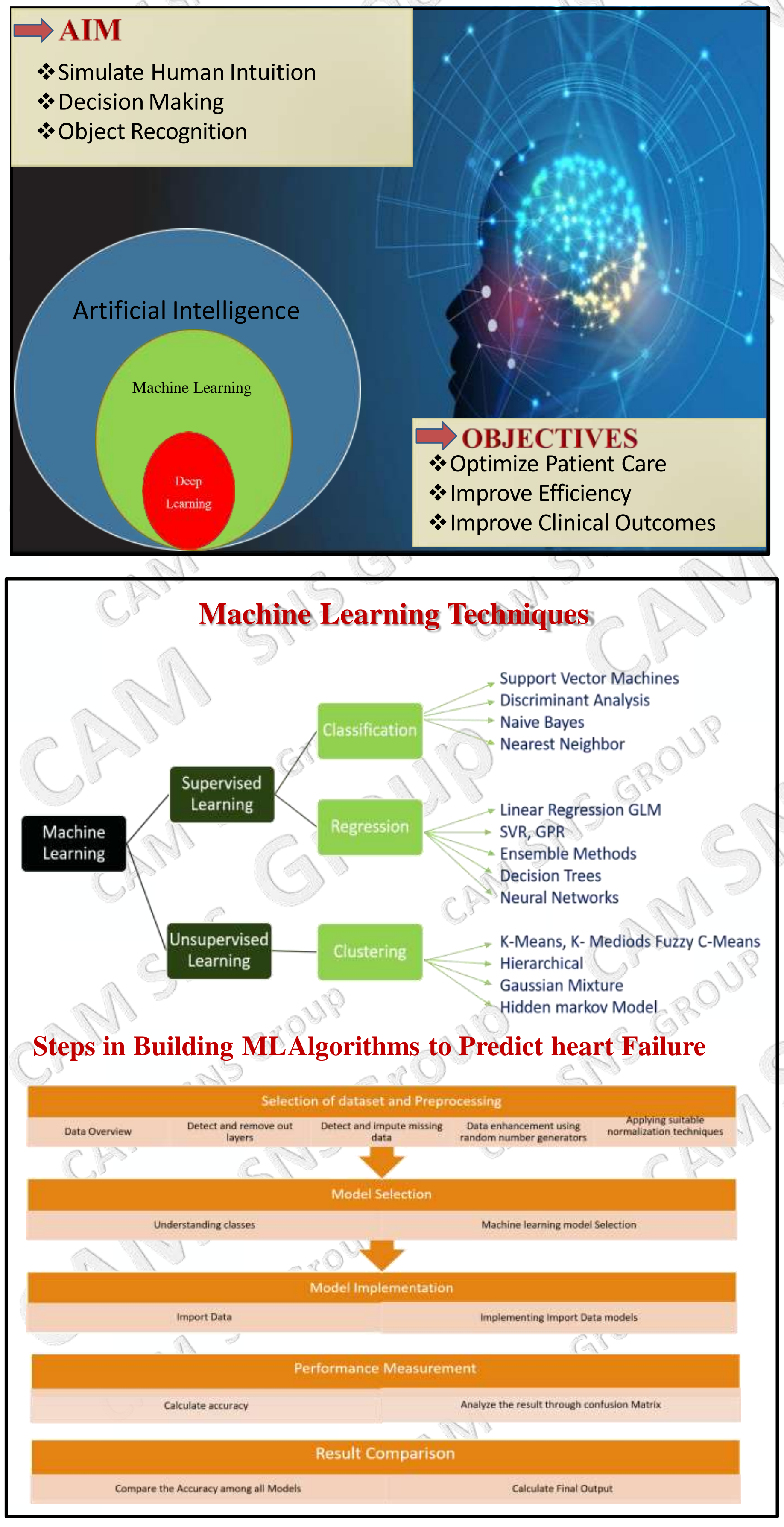

\section{Literature Review}

For a long time researchers have been working on identifying and predicting different diseases using machine learning.

\section{Number of Papers Published}

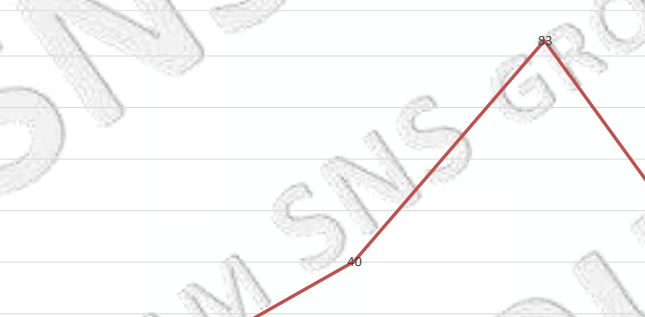

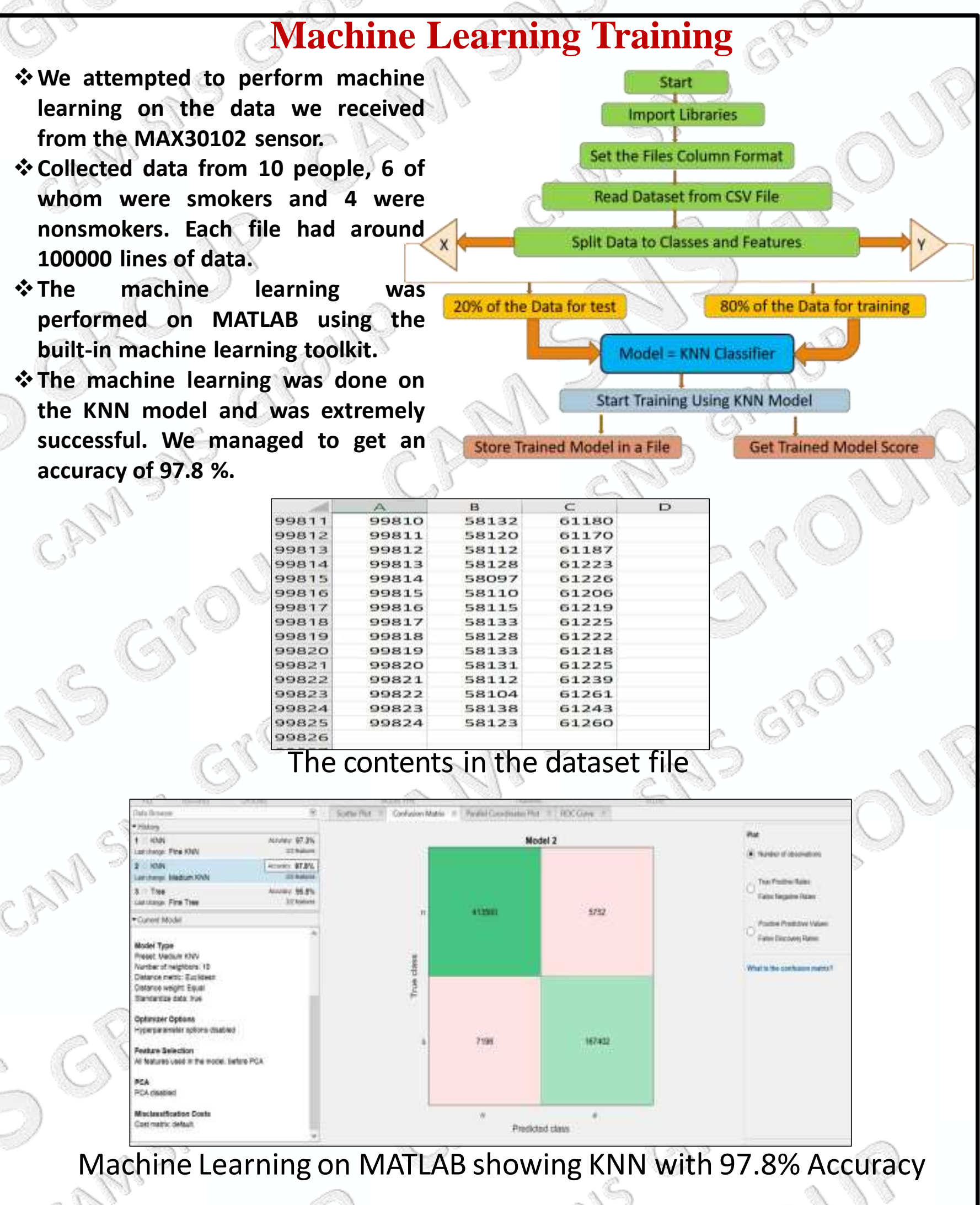

Conclusion

- Clinical decision-making is reliant on synthesizing high-quality data to help solve patient problems.

- As the amount of available patient data increases, it is likely clinical decision-making will be augmented by Al techniques in the future.

- There are a large variety of $\mathrm{ML}$ approaches and the most appropriate algorithm choice will be guided by the research question and the type of data available.

\section{Acknowledgement}

- This work was supported by Qatar University Internal Grant No. IRCC-2020-013 and Sultan Qaboos University through Grant \# $\mathrm{CL} / \mathrm{SQU}-\mathrm{QU} / \mathrm{ENG} / 20 / 01$, respectively. The findings achieved herein are solely the responsibility of the authors. 